www.jmscr.igmpublication.org

Impact Factor 5.84

Index Copernicus Value: 71.58

ISSN (e)-2347-176x ISSN (p) 2455-0450

crossref DOI: _https://dx.doi.org/10.18535/jmscr/v5i12.107

Journal Of Medical Science And Clinical Research

\title{
Prospective observational study of volume preloading prior to subarachnoid block in lower abdominal surgery with HETA starch (6\%) and Ringer Lactate solution
}

Authors

Ujjwala B Khairmode ${ }^{1}$, Padmanabha D V ${ }^{2}$, Ramdas Morale ${ }^{3}$

${ }^{1}$ Associate Professor, Department of Anesthesia, Government Medical College, MIRAJ

${ }^{2,3}$ Assistant Professor, Department of Anesthesia, Government Medical College, MIRAJ

Corresponding Author

Dr Padmanbha D V

Assistant Professor, Department of Anesthesia GMC, MIRAJ

Room no 6, I HR hostel, PVPGH Civil Hospital, Sangli, Maharashtra 416416

Email:dvpadmanabha@gmail.com

\begin{abstract}
We undertook a study to compare a colloidhetaethyl starch $6 \%$ with a crystalloid Ringer Lactate as preloading fluids for the prevention of sub arachnoid block induced hypotension.

Materials and Methodology: 100 patients were included in the study. Patients in Group $R$ received Ringer's lactate at $10 \mathrm{ml} / \mathrm{Kg}$ and Group $\mathrm{H}$ received $6 \%$ Heta starch at $10 \mathrm{ml} / \mathrm{Kg}$. Both these solutions were infused over a period of 20 minutes before performance of subarachnoid block. After preloading, all patients received Ringer's Lactate solution as maintenance fluid. Under aseptic precautions lumbar puncture was performed with 25 gauge spinal needle (Quincke's needle) through midline approach with patient in right lateral or left lateral decubitus position at third Lumbar intervertebral space. After free flow of CSF, 3.5 of $0.5 \%$ injection bupivacaine were injected. Immediately after the injection, the spinal needle is withdrawn and patient turned to supine position. Following Subarachnoid block, Systolic, Diastolic and Mean arterial blood pressure were recorded at following intervals -1,5,10,15,30,45,60,90 minutes and 3 hours after spinal anaesthesia. Sensory level of blockade was checked using pinprick method.

Results: Both groups were comparable with respect to preoperative pulse rate, blood pressure SBP and MAP without any statistically significant difference. There was a decrease in blood pressure in both the groups at 5 minutes and onwards after spinal anaesthesia. The difference was statistically significant at 10 min onwards. The decrease in blood pressure was maximum at $30 \mathrm{~min}$ in $R$ group and $60 \mathrm{~min}$ in group $H$. After that blood pressure was stable although it was less compared to the pre-operative. Overall decrease in blood pressure was statistically significant as compared to pre-operative blood pressure. The incidence of hypotension was double in group $R$ as compared to group H.The amount of mephenteramine required was $162 \mathrm{mg}$ in group $R$ and $60 \mathrm{mg}$ in group H.The total IV fluid requirement was more in group $R$ compared to group H.The incidence of decrease in pulse rate was almost similar in both groups but there was no bradycardia.Patients in group $R$ experienced nausea (2 patients) and vomiting (1 patient).

Conclusion: Hexa ethyl starch 6\% would be a better alternative to Ringer Lactate as a preloading fluid to prevent spinal induced hypotension.

Keywords: Preloading, Ringer lactate, Heta starch, subarachnoid block, hypotension.
\end{abstract}




\section{Introduction}

Spinal anaesthesia (SA) is used widely for elective and emergency surgeries as an anaesthetic technique of choice. ${ }^{1}$ It is popular over general anaesthesia because of its simplicity, cost effectiveness, use of minimal pharmacological agents, minimizes or avoids the problems of aspiration and difficult intubation. ${ }^{1}$ Patient also remains conscious throughout procedure so adverse effects easily assessed. It avoids neonatal drug depression from general anaesthesia in caesarean section. ${ }^{2}$ The operative blood loss is less. There is minimized risk of post-operative nausea and vomiting with adequate post-operative analgesia. It can be used in cases with respiratory tract infection which is usually a contraindication to general anaesthesia.

As having these advantages, the regional anaesthesia techniques including sub ara chnoid block has immensely attracted anesthesiologists. However, the common problems associated with SA are hypotension (most common), patient's cooperation is necessary high block, and deranged coagulation profile. ${ }^{3}$

The incidence of hypotension is reported to be around 20-80\% without institution of any prophylactic measures which can eventually cause nausea, vomiting and cardiovascular collapse. In order to achieve a satisfactory level of analgesia for lower abdominal surgeries, sensory block must extend up to T6. ${ }^{3,4}$ Due to increased capacity of intravascular space, patients often develop a relative hypovolemia during the procedure. ${ }^{5,6,7}$ Thus, protocols that aim to prevent this hypotension during SA may result in better outcomes than those designed to treat hypotension post occurrence. Prophylactic measures have been tried over the years to prevent or reduce this spinal induced hypotension, namely limiting the level of block up to desired level only by choosing ablock limited to suit the planned procedure preventing unnecessary decrease in arterial pressure, Mechanical / Pneumatic compression of lower limbs (Inflatable boots, elastic bandages, compression stockings Inflatable full length splints, Esmarch bandages), ${ }^{8-11}$ by lateral uterine displacement done by putting wedge or mechanical devices like sluderin obstetrics patients, ${ }^{12}$ prophylactic use of vasopressors (Mephenteramine, Dopamine, Phenylephrine, Ephedrine) $)^{13-15}$ and Preloading the circulation.

\section{Preloading the circulation}

Preloading prior to central neural blockade is one of the mainstays in the prevention of hypotension and use of a variety of volumes of crystalloids and colloids have been described in literature. Colloids have better intravascular expansion capability, less volume requirement and long duration of action in spite of high cost and fear of allergic reactions.

Following spinal anaesthesia, hypotension is commonly seen because of autonomic blockade which is 2 segments higher than the sensory block. To prevent hypotension pre loading is routine practice with Ringers lactate or N.S. but it is not very efficient in preventing hypotension as only $1 / 3$ of administered dose of RL or NS will remain in the intravascular compartment which may explain why hypotension associated with spinal anaesthesia cannot be completely eliminated by crystalloid preloading and also the value of preloading has been questioned from time and time again. Too much crystalloids also cause overloading of pulmonary circulation resulting in signs of over hydration, to prevent this, small volumes of colloid are tried. Heta starch $(6 \%)$ proved to be safe colloid and widely used in all situations.

Preloading might maximize intravascular volume expansion during vasodilation from the sympathetic blockade. Augmentation of blood volume with volume preload must be great enough to result in significant increase in cardiac output. In an effort to increase cardiac preload before instituting spinal blockade in the hope of preventing subsequent hypotension the concept of preload was born.

Preloading was first used by Wollman and Marx in 1968 in human subjects when they demonstrated that hypotension could be 
eliminated by use of one liter of D5RL before spinal block. ${ }^{16}$ Although intravenous fluid preload became an established part of anesthetic practice, it soon became apparent that it was not completely effective in preventing hypotension. However, it was assumed that, because the theory was sound, more preload was required in some patients.

Literature continues to emphasize the role of intravenous fluid loading as a best prophylactic measure in reducing the incidence of hypotension. After taking into consideration the preference for regional anesthetic techniques in the present era and along with it, the associated problem of hypotension, and in view of the controversy regarding crystalloid and colloid pre loading we undertook a study to compare a colloid- hexa ethyl starch $6 \%$ with a crystalloid - Ringer Lactate as preloading fluids for the prevention of sub arachnoid block induced hypotension for lower abdominal surgery.

\section{Materials and Methodology}

After obtaining approval from institutional ethics committee and written informed consent patients were recruited in the study. The study was undertaken in the Department of Anesthesiology, at a tertiary care centre from January 2015 to September 2016. The study was carried out in 100 patients of ASA physical status I and II of either sex, with age ranging from 20-60 years to be posted for elective and emergency lower abdominal surgeries.

Patients in ASA Grade III \& IV, below 12 years of age, pregnant patients, those with cardiovascular, respiratory and central nervous system and renal diseases and also those patients requiring supplementation of general anesthesia were excluded. Pre-anesthestic evaluation was done on the previous day of surgery with baseline investigations. A peripheral intravenous line with 18 gauze cannula was secured in one of the upper limb. Volume of infusion was determined according to body weight.

All patients were divided in two groups. Patients receiving Ringer's lactate at $10 \mathrm{ml} / \mathrm{Kg}$ were grouped as group-R and 6\% Heta starch at 10 $\mathrm{ml} / \mathrm{Kg}$ were grouped as group-H respectively. Both these solutions were infused over a period of 20 minutes before performance of subarachnoid block. After preloading, all patients received Ringer's Lactate solution as maintenance fluid. Baseline parameters and post spinal parameters were recorded using an electronic patient monitoring device. Lumbar puncture was performed with 25 gauge spinal needle (Quincke's needle) through midline approach with patient in right lateral or left lateral decubitus position at third Lumbar intervertebral space. After free flow of CSF, 3.5 of $0.5 \%$ injection bupivacaine were injected. Immediately after the injection, the spinal needle was withdrawn and patient turned to supine position. All patients in Group $\mathrm{R}$ and in Group $\mathrm{H}$ had sensory level block achieved around T6.

Following Subarachnoid block, systolic, diastolic and mean arterial blood pressure (MAP) were recorded at following intervals - 1,5,10,15,30, 45,60,90 minutes and 3 hours after spinal anaesthesia. Rescue medications wherever administered were recorded. Sensory level of blockade was checked using pin prick method.

Hypotension is defined as a decrease in the MAP by more than $25 \%$ from the baseline level and was managed by increasing fluid infusion rate and administering $100 \%$ oxygen by mask. If hypotension still persists, injection mephenteramine sulphate was administered intravenously $6 \mathrm{mg}$ bolus and repeated until the blood pressure increased to acceptable levels.

Bradycardia was defined as pulse rate less than 60 beats per minute. Bradycardia was treated with IV Atropine $0.6 \mathrm{mg}$.

\section{Results}

Patients were randomly allocated into two groups of 50 each. Group $\mathrm{H}(\mathrm{n}=50)$ received $10 \mathrm{ml} / \mathrm{kg}$ of hexa ethyl starch $6 \%$ as preloading fluid over 20 minutes. Group $\mathrm{R}(\mathrm{n}=50)$ received $10 \mathrm{ml} / \mathrm{kg}$ of Ringer Lactate as preloading fluid over 20 minutes. The mean age in Group $\mathrm{H}$ was $37.32 \pm$ 
9.74 years and in Group R was $38.8 \pm 10.8$ years. The male: female ratio was $1: 1$ in both groups. Body weight of the patients varied from $40-80 \mathrm{~kg}$ with mean weight in Group $\mathrm{R}$ and $\mathrm{H}$ being $59.52 \pm 8.36$ and $59.08 \pm 7.86$ respectively. In majority of the patients preoperative pulse was between $80-100$ with mean values of $89.42 \pm 5.89$

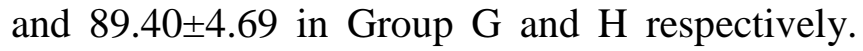
All the patients had their pre-operative blood pressure in the normotensive range. Majority of patients 48 patients in Group $\mathrm{R}$ and 50 patients in Group $\mathrm{H}$ had pre-operative systolic blood pressure between 112- $130 \mathrm{~mm} \mathrm{Hg}$. 2 patients in Group $\mathrm{H}$ had systolic blood pressure up to $140 \mathrm{~mm} \mathrm{Hg}$. This higher blood pressure could again be attributed to pre-operative anxiety due to surgical procedure. The mean baseline systolic blood pressure was almost comparable in both the groups without any statistically difference ranging from $112-130 \mathrm{mmHg}$ with mean values of $120.64 \pm 5.44(\mathrm{G})$ and $120.88 \pm 4.70(\mathrm{H})$ and also mean of mean arterial blood pressure were 91.64 $\pm 3.92(\mathrm{G})$ and $91.78 \pm 3.92(\mathrm{H})$.

The patients undergoing various surgical procedures in both the groups, in group R, 16 patients had operated as abdominal hysterectomy, 21 as appendectomy and 13 as inguinal hernia. Where as in Group H, 15 patients had operated as abdominal hysterectomy,19 as appendectomy and 16 as inguinal hernia repair. Considering procedures in both the groups it was almost same in both the groups. The mean duration of surgery in both groups were $89.28 \pm 4.49$ (G) and $88.6 \pm 4.69(\mathrm{H})$.

Table 1 shows changes in mean pulse rate at various time intervals in both the groups

\begin{tabular}{|l|c|c|}
\hline & Group R & Group H \\
\hline Pre op & $89.42 \pm 5.89$ & $89.04 \pm 4.69$ \\
\hline $1 \mathrm{~min}$ & $88.28 \pm 5.56$ & $88.82 \pm 4.44$ \\
\hline $5 \mathrm{~min}$ & $85.86 \pm 4.44$ & $86.08 \pm 4.47$ \\
\hline $10 \mathrm{~min}$ & $84.48 \pm 4.27$ & $84.78 \pm 4.29$ \\
\hline $15 \mathrm{~min}$ & $82.16 \pm 11.49$ & $82.42 \pm 11.50$ \\
\hline $30 \mathrm{~min}$ & $83.68 \pm 4.59$ & $83.74 \pm 4.08$ \\
\hline $45 \mathrm{~min}$ & $83.74 \pm 4.27$ & $84.04 \pm 3.95$ \\
\hline $60 \mathrm{~min}$ & $83.76 \pm 4.26$ & $84.08 \pm 3.79$ \\
\hline $90 \mathrm{~min}$ & $83.88 \pm 4.34$ & $84.20 \pm 3.87$ \\
\hline $3 \mathrm{HRs}$ & $82.38 \pm 11.88$ & $84.26 \pm 11.80$ \\
\hline
\end{tabular}

There was a decrease in pulse rate in both the groups at 5 minutes and onwards after spinal anaesthesia. The difference was statistically significant. The decrease in pulse rate was maximum at $15 \mathrm{~min}$ in both groups. After $30 \mathrm{~min}$ pulse rate was stable although it was less compared to the pre-operative. Overall decrease in pulse rate was statistically significant as compared to preoperative pulse rate.

Table 2 shows changes in mean systolic blood pressure and MAP at various time intervals in both groups.

\begin{tabular}{|l|c|c|c|c|c|c|}
\hline & \multicolumn{2}{|c|}{ Group R } & \multicolumn{2}{c|}{ Group H } & \multicolumn{2}{c|}{ P value } \\
\hline & $\begin{array}{c}\text { Mean } \\
\text { SBP } \pm \text { SD }\end{array}$ & MAP & $\begin{array}{c}\text { Mean } \\
\text { SBP } \pm \text { SD }\end{array}$ & MAP & $\begin{array}{c}\text { Mean } \\
\text { SBP } \pm \text { SD }\end{array}$ & MAP \\
\hline Pre op & $120.64 \pm 5.44$ & $91.64 \pm 3.92$ & $120.88 \pm 4.7$ & $91.78 \pm 3.92$ & 0.0 .81 & 0.85 \\
\hline $1 \mathrm{~min}$ & $118.92 \pm 6.7$ & $90.45 \pm 4.63$ & $119.6 \pm 5.35$ & $91.06 \pm 4.37$ & 0.57 & 0.49 \\
\hline $5 \mathrm{~min}$ & $114.08 \pm 6.88$ & $86.27 \pm 4.53$ & $116.08 \pm 4.96$ & $87.44 \pm 4.42$ & 0.0986 & 0.19 \\
\hline $10 \mathrm{~min}$ & $110.32 \pm 6.87$ & $83.52 \pm 4.38$ & $113.20 \pm 5.44$ & $84.61 \pm 4.23$ & 0.0221 & 0.20 \\
\hline $15 \mathrm{~min}$ & $105.96 \pm 6.59$ & $80.68 \pm 3.99$ & $110.4 \pm 5.54$ & $82.29 \pm 4.05$ & 0.0004 & 0.048 \\
\hline $30 \mathrm{~min}$ & $100.92 \pm 7.8$ & $77.29 \pm 3.99$ & $105.8 \pm 7.88$ & $79.18 \pm 3.94$ & 0.0024 & 0.004 \\
\hline $45 \mathrm{~min}$ & $101.92 \pm 5.19$ & $76.07 \pm 2.82$ & $104.12 \pm 5.47$ & $77.38 \pm 3.59$ & 0.0417 & 0.086 \\
\hline $60 \mathrm{~min}$ & $102.20 \pm 5.13$ & $76.95 \pm 3.61$ & $104 \pm 5.13$ & $77.46 \pm 3.56$ & 0.0824 & 0.47 \\
\hline $90 \mathrm{~min}$ & $106.68 \pm 5.07$ & $81.12 \pm 3.48$ & $108.4 \pm 4.96$ & $81.60 \pm 3.47$ & 0.0895 & 0.49 \\
\hline $3 \mathrm{hr}$ & $109.92 \pm 5.19$ & $84.31 \pm 3.76$ & $111.6 \pm 4.61$ & $84.72 \pm 3.57$ & 0.0901 & 0.57 \\
\hline$* p<0.05-j u s t$ significant, $* * p<0.01-$ moderately significant, $* * * p<0.001-$ highly significant \\
\hline
\end{tabular}

There was a decrease in blood pressure in both the groups at 5 minutes and onwards after spinal anaesthesia, maximum at $30 \mathrm{~min}$ in $\mathrm{R}$ group and
$60 \mathrm{~min}$ in group $\mathrm{H}$. The difference was statistically significant at 10 min onwards. 
The no of patients requiring mephenteramine were double in group $\mathrm{R}$ (20 patients) as compared to group $\mathrm{H} \quad(10$ patients $)$. The incidence of hypotension was also double in group R (40\%) as compared to group $\mathrm{H}(20 \%)$. The amount of mephenteramine required was $162 \mathrm{mg}$ in group $\mathrm{R}$ and 60mg in group $H$ with $p$ value 0.003, statistically significant for need in Group R.

The total IV fluid requirement was more in group $\mathrm{R}$ (mean 1017.8 $\pm 94.91 \mathrm{ml}$ ) compared to groupH (mean 915.6 $\pm 80.17 \mathrm{ml}$ ). The difference in fluid requirement by the two groups was statistically significant ( $\mathrm{P}$ value -0.0000001)

\section{Complications}

The incidence of decrease in pulse rate was almost similar in both groups but there was no bradycardia. The incidence of hypotension was twice $(40 \%)$ in group $\mathrm{R}$ compared to group $\mathrm{H}$ (20\%). 2 patients in group $\mathrm{R}$ had nausea compared to 1 in group $H .1$ patient in group $R$ had vomiting. No patient had bronchospasm or severe anaphylactic reaction in both Groups.

\section{Discussion}

Currently regional anaesthesia is preferred because of its simplicity, reliability, decreased incidence of complications due to aspiration and endotracheal intubation and cost effectiveness. However, hypotension still remains the most common problem associated with spinal anaesthesia. There is a definite correlation between the dermatomal level and incidence of hypotension and bradycardia.

The major factor in the development of hypotension is the level of the block. The sympathetic outflow is between $\mathrm{T} 1$ and L2, so that a block below that level has no effect on arterial pressure, but one to T8 sympathectomizes the lower half of the body. The sympathetic supply to the adrenal medulla is from T8 to L1 and central neural blockade alters the arterial pressure by inhibition of systemic release of catecholamines. Fibers from $\mathrm{T} 2$ to $\mathrm{T} 4$ provide the sympathetic supply to the heart and decreased contractility and bradycardia ensues if they are blocked. A block as high as $\mathrm{T} 1$, totally removes the ability of the body to compensate for other circulatory changes in addition to producing excessive vasodilation. The level of sympathetic block is in general higher than sensory block, because a high level of block is a major determinant of development of hypotension, choosing a block limited to suit the planned procedure prevents unnecessary decrease in arterial pressure. Overall, the patients with anesthetic levels of T4 and higher had a significantly greater incidence of hypotension, than those patients with dermatomal levels below T4.

In our study, there was a decrease in pulse rate in both the groups at 5 minutes and onwards after spinal anaesthesia which was statistically significant $(\mathrm{P}<0.05)$ and maximum fall at 15 minutes. After 15 minutes the pulse rate was stable although it was less as compared to the preoperative pulse rate. Overall the pulse rate in the intraoperative and postoperative period was less than the preoperative pulse rate. The decrease was not to the limit of defined bradycardiain both the groups requiring treatment with atropine was nil. A cause of fall in pulse is the lowering of the blood pressure in the right atrium consequent on diminished venous return (Bainbridge effect) and also may be secondary to a gradual spread of the local anaesthetic to involve the cardiac sympathetics. Our findings were comparable with Baraka A. S. et al and Sharma S.K. et al who found that the mean control heart rate values were similar in both the groups and did not show any significant change after intravascular administration. ${ }^{17,18}$

There was a decrease in systolic blood pressure and MAP in both the groups at 5 minutes and onwards after spinal anaesthesia which was statistically significant $(\mathrm{P}<0.05)$ after 10 minutes and fall was maximum at 30 minutes in both the groups but the fall was more in Ringer Lactate group. The decrease in the blood pressure value and MAP noted in the mean intraoperative as well as postoperative readings, when compared to the preoperative blood pressure was also statistically 
significant $(\mathrm{P}<0.001)$. Thus, the number of patients requiring mephenteramine was more than double in Ringer Lactate group (Group R) as fall in mean arterial blood pressure below the $75 \%$ of pre op level.

Considering the higher incidence of hypotension in Ringer Lactate group, the overall fluid requirement to maintain blood pressure was more in Ringer Lactate group. These results were comparable to those of Imran sheikh et al and Singh B el al. that hydroxy ethyl starch is more effective than lactated Ringer's solution as a pre loading fluid in prevention of hypotension following SA, and incidence of hypotension was only reduced but could not be completely eliminated.

Sharma S.K et al found that the incidence of hypotension was significantly greater in Lactated Ringer solution group than in Hetastarch group $\{[11 / 21(52 \%)$ versus $3 / 19(16 \%)] ; \mathrm{P}<0.05\} .{ }^{18}$ Rao N.S. et al also found that incidence of hypotension in crystalloid group was $52 \%$ and in colloid group was $27 \%$. The peak fall in blood pressure occurred within 2-5 minutes in both the groups. ${ }^{19}$ The average consumption of mephenteramine in crystalloid group was $12 \%$, while in colloid group was only $2 \%$.

Lt. Col. Jaitwat et al in their study found that the incidence of hypotension in crystalloid group was $45 \%$ and in the colloid group was $25 \% .{ }^{20}$ The peak fall in blood pressure was recorded within 2-5 minutes in both the groups. The average consumption of mephenteramine in crystalloid group was $15 \%$ compared to $4 \%$ in colloid group.

French G.W.G. et al in 1999 in their study observed that more patients in the Hartmann's group $(\mathrm{n}=38)$ developed hypotension compared with Pentastarch group $(\mathrm{n}=10)(\mathrm{P}<0.0001) .{ }^{21}$ The mean dose requirement of ephedrine was also higher in Hartmann's group $(\mathrm{P}<0.001)(8.35 \mathrm{mg}$ versus $1.35 \mathrm{mg}$ ), thus crystalloid preloading does not eliminate the incidence of hypotension or ephedrine requirements after SA.

Thus our study also showed colloid preferable than crystalloid. Crystalloid solutions have less viscosity so can be infused rapidly, but have extremely short intravascular half life and are required to be infused in large volumes, which can be hazardous. On the contrary, Colloid solutions contain polymerized macromolecules and different concentration of ions, relatively impermeable to the capillary membrane. Colloids have the ability to restore blood volume and offset hypotension and hypovolemia more effectively when used as replacement fluids. Colloids maintain colloid osmotic pressure, thereby preventing the development of pulmonary oedema and decreases the need of vasopressors.

Nausea and vomiting may cause much misery to the conscious patient under SA, possible causes are afferent impulses along the unblocked vagus, increased peristalsis, back ward flow of bile into the stomach through the relaxed sphinder of oddi and pylorus, acute hypotension, cerebral hypoxia, drugs used for premedication and psychic factors. All the colloid volume substitutes carry the risk of anaphylactoid reactions due to a nonspecific release of histamine with a total incidence of $0.033 \%$, the frequency of life threatening reactions being $0.003 \%$ for plasma protein solutions. $0.006 \%$ for hydroxyethyl starch $0.008 \%$ for Dextran $0.038 \%$ for Gelatinsolutions. ${ }^{22}$ Hexa ethyl starch 6\% may also cause these reactions in varying severity, ranging from simple skin symptoms like urticarial to much less frequent but more severe reactions involving acute decrease in blood pressure, shock, bronchospasm, cardiac or respiratory distress which may occur both in conscious or anaesthetized patient. ${ }^{23}$

The patient should be carefully monitored during the infusion, particularly during the administration of first 20-30 ml. If there are signs of side effects, the infusion must be stopped immediately. The further therapeutic steps should depend on the clinical severity. By early diagnosis, prophylaxis and treatment, the prognosis of anaphylactoid reactions in general is good; "The earlier the treatment, the better the outcome." 


\section{Conclusion}

Preloading protects the blood vessels from the effects of relaxation and the hydrostatic pressure maintains the hemodynamics. Volume preloading has emerged as the single most important and effective way to prevent hypotension and its consequences. Irrespective of whether crystalloids or colloids are used for preloading the circulation before spinal anesthesia, hypotension is not totally eliminated. But, owing to the property of colloids being retained longer in the vascular space while rapid leak extravascularly of crystalloids, degree and incidence of hypotension and requirement of vasopressors is reduced with colloids. Further improved haemodynamic stability is maintained with colloids. Taking the results and minimum side effects into consideration it can be concluded from the above study, that hexa ethyl starch $6 \%$ would be a better alternative to Ringer Lactate as a preloading fluid to prevent spinal induced hypotension.

\section{References}

1. Greene N M. The physiology and spinal anaesthesia. 3rd edition, Baltimore : Williams and Wilkins, 1981;63;146, 26573

2. Gunusenet al.Effects of fluid preload compared with crystalloid co-load plus ephedrine infusion on hypotension and neonatal outcome during spinal anesthesia for caesarean delivery. Anesth intensive care.2010 Jul;38(4):647-53

3. Hallworth D, Jellicoe J A, Wilkes R G. Hypotension during epiduralanaesthesia for caesrean section. Anaesthesia 1982; 37: 53-6

4. Thorburn J, Moir D D. Epidural analgesia for elective caesarean section. Technique and its assessment. Anaesthesia 1980;35: 346

5. Murray A M, Morgan M ,Whitmann J. Crystalloid versus colloid forcirculatory preload for epidural caesarean section. Anaesthesia 1989;44 :463-6
6. Sathe U, Shah B N. Spinal preloading Colloids versus crystalloids. BullIntensive Crit Care 1996; 3:4-15

7. Shapira S C ,Shir Y, Shapira Y A. Comparison of intravenous loadingfor epidural anaesthesia with Ringers Lactate or polygeline solutions. European J Pain 1991; $12: 100-3$

8. Bhagwanjee S, Rocke D A, Rout C C et al.:Prevention of hypotension following spinal anaesthesia for elective caesarean section by wrapping of the legs. British Journal of Anaesthesia 1991;65:819-22

9. Gibbs C P ,Werba J V, Banner T E et al. Epidural anaesthesia :legwrapping prevents hypotension. Anaesthesiology 1983; 59 : A405

10. Gondie T A , Winter W, Fergusson D J M. Lower limb compression using inflatable splints to prevent hypotension during spinal anaesthesia for caesarean section. Acta Anaesthesiologica Scandinavica 1988; $32: 541-4$

11. James F M III, Greiss F C, Kenup R A. An evaluation of vasopressor therapy for maternal hypotension during spinal anaesthesia. Anaesthesiology 1970; 33 : 25-34.

12. Kennedy R L. An instrument to relieve inferior vena cava occlusion.AmJ Obstet Gynecol. 1970;107; 331-3

13. Cucchiara R F, Rest all C J. Mephenteramine and intravenous fluidsfor the prevention of hypotension associated with spinal anaesthesia. Anaesthesiology 1973; $39: 109-10$

14. Buggy, Brunner JA. Dopamine for the treatment of spinalhypotension during caesarean section. Anaesthesiology 1980;514-7

15. Hemmingsen C, Poulson J A, Risbo A. Prophylactic ephedrine duringspinal anaesthesia : double blind study in patients in ASA groups 1-3.British Journal of Anaesthesia 1989; 63 : 340-2 
16. Wollman S B. Marx G F. Acute hydration for prevention of hypotension of spinal anaesthesia in parturients. Anaesthesiology 1968; 29:374-80

17. Baraka A S, Taha S K, Ghabach M B, A Abla, Sibaii N, Nader A M. Intravascular administration of polymerized 31940elatine versus isotonic saline for prevention of spinal induced hypotension. Anesthesia Analgesia 1994;78:301-5

18. Sharma S K , Gajraj N M , Sidawi J E. Prevention of hypotension during spinal anaesthesia. A Comparison of intravascular administration of Hetastarch versus Lactated Ringer solutions. Anesthesia Analgesia 1997; 84 ; 111-4.

19. Rao N S , Vijayalaxmi P R, Singh T P D. Crystalloid versus colloid preload for preventing hypotension in caesarean section under spinal anaesthesia IJIM. 1997; $8: 66-9$

20. Lt. Col. Jaitwat, Col. Y V Suri. Comparative evaluation of Ringer Lactate and $3.5 \%$ Polygeline (Haemaccel) solution as preloading fluid for prevention of hypotension following spinal anaesthesia.

21. French G W G, White J B, Howell S J et al. Comparison of Pentastarch and Hartmann's solution for volume preloading in spinal anaesthesia for elective caesarean section. British Journal of Anaesthesia 1999; $83: 475-7$

22. Singh N R, Singh K H, Singh N T. : Anaphylactoid reaction to modified fluid elatine. Indian. J. Anaesthesia 2001; 45 : 222-3

23. Singh et al: Ringer lactate vshexa starch: Comparative study of preloading with ringer lactate $\mathrm{v} / \mathrm{s}$ 6\% hexa starch solutions to prevent hypotension following spinal anaesthesia in elective surgery. 1178-81. 